\title{
Genetic Drift and Polygenic Inheritance
}

FRANK B. LIVINGSTONE

Department of Anthropology, University of Michigan, Ann Arbor, Michigan 48104

KEY WORDS Simulation - Polygenic Inheritance $\cdot$ Optimizing

Selection - Genetic Drift - Metric Traits.

\begin{abstract}
The interaction of random gene drift and selection was studied by computer simulation for two quantitative traits, which were considered to approximate stature and skin color differences in human populations. The expected effects of gene drift, fixation of alleles and reduction of genotypic and phenotypic variances, were found in the simulation. Stabilizing selection, which seems to be the type of selection operating on these traits, was found to increase the effects of gene drift. Since there seems to be no evidence of reduction in phenotypic and presumably genotypic variability in small human populations, the applicability of these simple genetic models to human traits raises problems for which several possible solutions exist.
\end{abstract}

Ever since the development of the theory of population genetics over 50 years ago, its implications for the variability in metric traits among human populations has been a continuing problem. Most of the concepts of population genetics pertain to traits controlled by a single locus with a limited number of alleles, while most anthropometric traits are influenced by several loci and also by the environment. These complications must obviously be considered in any interpretation of the effects of mutation, selection, gene flow, and gene drift on metric traits. In particular, random genetic drift is known to change gene frequencies significantly in small populations, but the question remains as to its effect on anthropometric traits. Some time ago Birdsell (50) pointed out that, assuming the common polygenic model of equal and additive effects for all loci, one would expect very little change in the mean of any trait since the alleles increasing its size would be expected to increase and become fixed in the population as frequently as those decreasing the trait. However, selection would presumably have some effect on this process of drift, and its effect would obviously depend on how selection operated on the trait.

Beginning with the classic paper by Fisher ('18) on the correlation of metric traits among relatives, population genetics theory has been applied to quantitative inheritance. The theory has been developed from and widely used in animal and plant breeding, but there have been few applications to the problems of human evolution. To a great extent this is obviously due to a lack of knowledge of both the mode of inheritance of anthropometric traits and the action of natural selection on them, but I think it is also due to a lack of confidence in the applicability or usefulness of the simple models of quantitative inheritance. Certainly the concept or assumption of equal and additive loci is a crude approximation and the number of loci affecting most metric traits is not known, but the importance of these assumptions to the evolutionary process will only become known through a comparison of our crude models with data.

The models of quantitative inheritance are also so complex that it has only been since the development of the computer that any attempt could be made to answer some of the questions. Formal solutions are frequently not possible, but simulations with various sets of numerical estimates for the many parameters can provide some idea of the effects of the various forces of evolution. A great many simulation programs have been developed to study aspects of genetic change in other organisms, particularly Drosophila. Young ('66, '67), Hill ('69), and Frankham et al. 
(68) are some recent attempts to study the interaction of selection and drift. For the most part these studies have used a model of directional selection with truncation. In this model there is a threshold value for the phenotype, and, depending on the direction of selection, all organisms on one side of the threshold value are allowed to breed but none on the other side. Although this is the usual procedure in animal breeding, it is obviously not the usual mode of operation of natural selection in human populations. Recent data for some metric traits in man have begun to show that selection most often operates according to some optimization or stabilization scheme (for birthweight, stature, and cephalic index, see Bajema (71) for the most recent studies). In each case an intermediate phenotypic value close to the mean has the highest fitness, and fitness decreases continuously as the phenotypic value diverges from this optimum value. The most generally accepted model assumes this decrease to be proportional to the square of the difference between the phenotype and the optimum value, but a linear or exponential decrease has been suggested for various traits. In this paper fitness is assumed to decrease with the square of the difference. Aspects of stabilizing selection have been studied in other organisms (Latter, '70; Latter and Novitski, '69; Scharloo et al., '67; Curnow, '64; Prout, '62), and the results are comparable to those reported here.

\section{METHODS}

The computer simulation represents the population by a $\mathrm{N} \times \mathrm{M}$ matrix, which classifies the $\mathrm{N}$ individuals as homozy. gotes for the large or small allele or heterozygotes at each of the $M$ loci. The program randomly selects two individuals with replacement from the population and then mates them separately for each of the $M$ loci. A single offspring is produced and is either stored or discarded according to a randomly determined scheme of optimizing selection. There is no compensation for individuals selected out. Sex, family size, and other variables which are known to affect the effective size of the population have not been considered in order to make the results comparable to the simplest model of genetic drift for a single locus for which the rate of decay of genetic variability is $1 / 2 \mathrm{~N}$. The fitness of the optimum phenotype is 1.0, and the fitness of any genotype with a phenotypic value, $W$, is: 1 - FIT(WOPT $-W)^{2}$, where WOPT is the phenotypic value of the optimum genotypes and the constant, FIT, is used to adjust the total amount of selection.

The loci have been assumed to have equal and additive effects. Thus, for the ith locus there is a plus and a minus allele, $A_{i}$ and $a$, respectively, and three genotypes, $A_{i} A, A a_{i}$, and $a_{i} a_{i}$, which contribute $2,1+D$, and 0 units, respectively, to the phenotype. $\mathrm{D}$ is a measure of dominance and can be,+- , or 0 depending on whether there is dominance for the + or - allele or no dominance. For any particular anthropometric trait the phenotypic expression can be approximated by a linear function of the sum of the effects at each locus as given above. The model does not include the environmental component, although this can easily be incorporated into any simulation (e.g., Young, '66). This is because the environmental component for metric traits can be considered to introduce error or "noise" in the process of evolution and thus to decrease the effects of the various forces. Since the major purpose of these simulations was to determine the direction of the forces of evolution, the results can be considered to be maximum effects; and any environmental component would tend to reduce them approximately in proportion to the heritability.

At all loci the allele frequencies were started at 0.5 and were run for 100 generations. Population size, total amount of selection, and the degree of dominance have been varied. For each set of these parameters ten replications were done in order to determine the variability of the response. The standard deviations on tables 1 and 2 have been computed from these ten replications.

\section{RESULTS}

Table 1 shows the results of two sets of parameters for a trait which is determined by four loci. The phenotypic expression 
is a crude attempt to reflect the differences in skin color among human populations. It assumes that all differences in skin color are due to two alleles at these four loci, such that an individual homozygous for all plus or "dark" alleles would have a phenotypic value of 20.0 , which is about the darkest human skin pigmentation as measured by percentage reflectance $(20 \%)$ of the red wave length (Livingstone, '69, has a more detailed review of the data and the model). An individual homozygous for all "light" alleles would have a value of 60.0 . The median value would thus be 40.0 , and this value has been assumed to be the optimum phenotype in table 1. Two loci have been assumed to have the light allele dominant, and two, the dark one, and this dominance has been set at $\mathrm{D}=$ 0.8 . The formula for determining the phenotype is: $20.0+5.0$ [ (no. of light alleles) +0.8 (no. of heterozygous loci with light allele dominant) - 0.8(no. of heterozygous loci with dark allele dominant)] .

It can be seen on table 1 that there is very little change in the mean value of the phenotype after 100 generations even with a population size of 50 , which is about the smallest human isolate, and regardless of the amount of selection. There is more variability among replicates with smaller population sizes, but nevertheless the mean remains between $35 \%$ and $45 \%$, which is a very small range compared to the total human range. The mean allele frequency also does not change appreciably, although over onehalf of the loci become fixed in the ten replicates. On the other hand the phenotypic variability decreases significantly as one would expect with increasing fixation and homozygosity of the loci. For most human populations the standard deviation of this measure of skin color is about $3-5 \%$, so that if this is a reasonable model of skin color inheritance, there should be a considerable amount of homozygosity in human populations. Populations at the extremes of the skin color spectrum would also be more homozygous and have less variance than those in the middle. However, there are numerous complications in the relationship of additive gene action and this reflectance per- centage (Harrison, '71). Nevertheless there are extreme variations in the variance for skin color reported in different populations, but there seems to be little, if any, correlation between variance and extreme mean values.

While the phenotypic variability decreases due to drift, as expected the genetic variability increases. With the smaller population size, there is more fixation of alleles as expected, but since there is also less selection for this run, this may be a contributing factor. However, as will be seen on table 2, stabilizing selection actually increases the rate of fixation. In any case, this model seems to imply that genetic drift could result in a considerable amount of homozygosity for a trait such as skin color which is controlled by approximately four loci if the population size has been close to the lower limits of the human species.

In order to determine the effect of the number of loci on the process of drift with selection, several different runs were made with ten loci determining a trait with the approximate distribution of stature in human populations. Of course, the number of loci controlling stature is not known with any certainty, but Penrose ('69), by an analysis of the correlations among relatives, has estimated that six loci are involved. Spuhler ('68), by another method, estimated that as few as six major loci could be contributing to differences in IQ scores, so that the possibility exists that fewer genes are involved in polygenic inheritance than is generally considered to be the case. No dominance has been assumed for most runs. Although Fisher's original work seemed to indicate almost complete dominance for stature, Barrai et al. ('64) could find no evidence for it. The mean stature was assumed to be 5.7 feet, and the range for the trait, to be from 4.8 feet to 6.6 feet. These extremes would be the phenotypes of the two completely homozygous genotypes for the minus or plus alleles. The formula for the phenotype is thus: $4.8+0.09$ (no. of plus alleles).

Table 2 shows the results of 12 runs of ten replicates each. Again there is no systematic change in either the mean phenotype or the average gene frequency, and the drift in mean stature is as great 


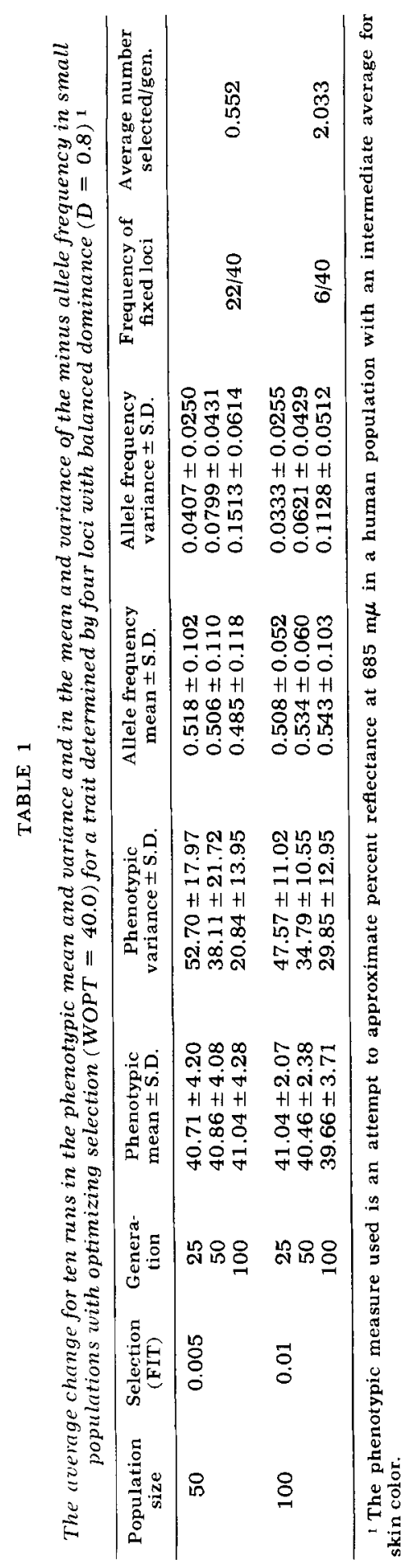

in the large populations as in the small ones. This occurs despite the fact that there is much more fixation of alleles in the small populations. On the other hand, the variability among replications seems to be smaller in the large populations, but this variability is also influenced by selection. The maximum amount of selection (FIT $=0.01$ ) results in slightly less than $4 \%$ of each generation being selected out, which seem minimal, but it does seem to decrease significantly the amount of drift around the mean value. With a population of 100 and no selection the average standard deviation is about 0.17 after 100 generations of evolution, and this would appear to be close to equilibrium. The range is thus about 0.7 foot, so an individual population could diverge from the mean by up to four inches, which seems rather large.

As expected, the phenotype and gene frequency variances were significantly changed during the simulation. The phenotypic variance declined, and this decline was inversely proportional to the population size. Figure 1 shows the decline in phenotypic variance for the runs shown on table 2. Several runs were made with dominance $(\mathrm{D}=0.8)$, and they fall within the range of the runs with no dominance. This indicates that dominance does not seriously affect the decline in phenotypic variance. It can be seen that after 100 generations all of the runs with a population of 25 have a greater decrease than other muns, and all those with a population of 50 decrease more than those with 100. Since at all loci the initial allele frequencies were 0.5 , the initial phenotypic variance was about 0.0425 . Thus, the decline is not linear but rapid at first with a leveling off.

The average standard deviation for human populations for stature is $5.81 \mathrm{~cm}$ (Howells, '36), which would correspond to a phenotypic variance of 0.0363 foot on our scale. Howells found a range in the standard deviation from 3.8 to $7.8 \mathrm{~cm}$, or 0.0156 to 0.0655 on our scale. This range encompasses most of the values on figure 1, although the upper limit is considerably greater than the maximum with ten loci. If the same range of variation is assumed to be due to more loci, the maximum variance would be even smaller; 


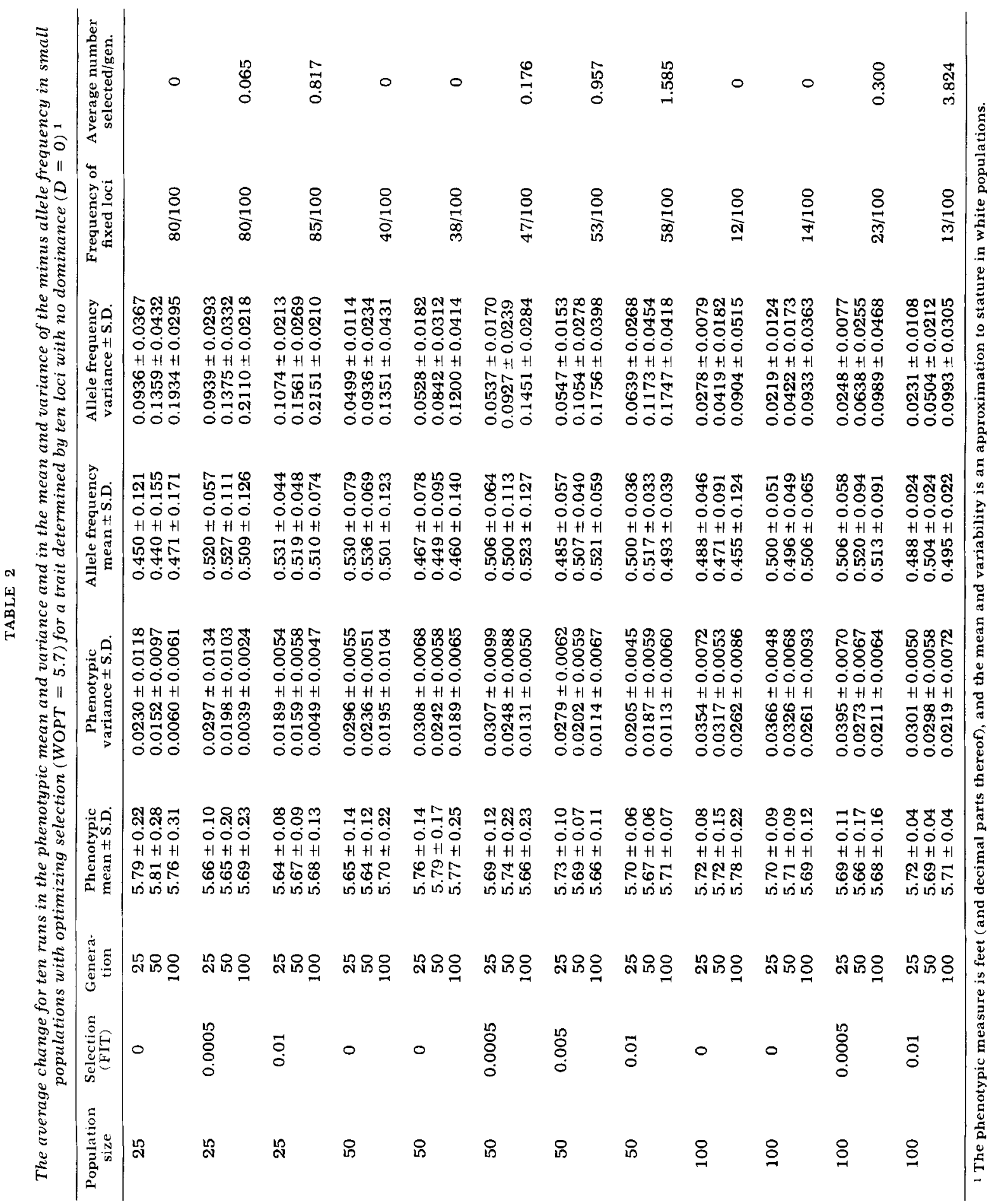




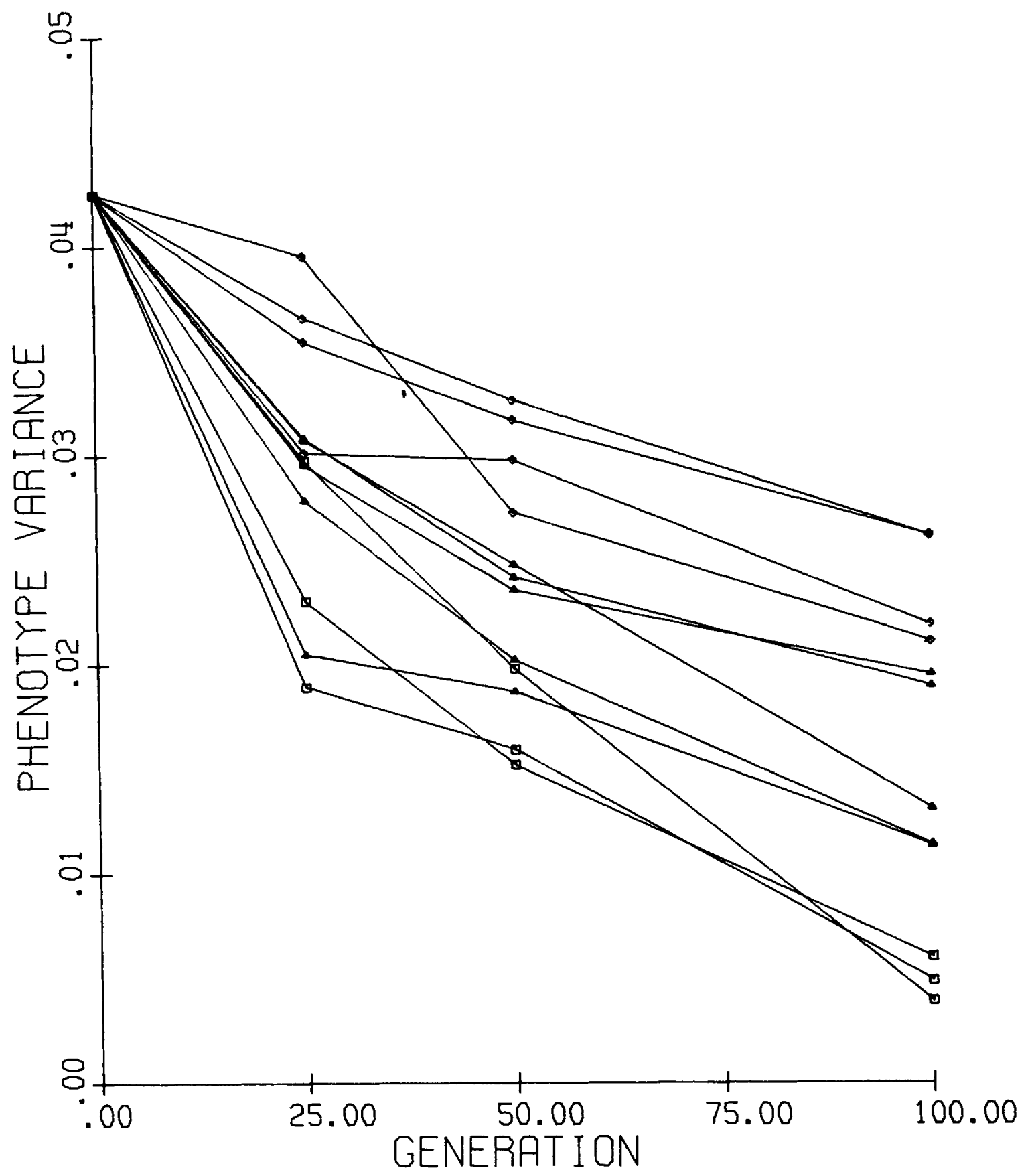

Fig. 1 The decrease in phenotypic variance with different population sizes and with different amounts of optimizing selection. The runs shown here are the same as those in table 2. Population $=25(\square \longrightarrow \square),=50(\Delta-\Delta),=100(\diamond-\diamond)$. 
for 20 loci it would be 0.02 and for 40 loci about 0.01 . If the heritability of stature is assumed to be 0.5 , which would be a minimum estimate, one-half of the total variance should be due to genetic differences. For the average population, this would be about 0.018 , which could be due to ten loci with a significant amount of homozygosity or 20 loci with almost the maximum amount of heterozygosity. However, despite the great variation in the standard deviation of stature among human populations, there seems to be no correlation with population size. The studies cited in Howells (36) and Trevor (53) do not show any trend, and the hybrid populations in the latter study also do not seem to be more variable. More recent data from a small isolated tribe in West Africa (Gomila, '71) and from the island of Tristan da Cunha (Marshall et al., '71) show standard deviations larger than the average. The population of Tristan da Cunha has an extremely high standard deviation; it is one of the most highly inbred of all human populations, and, given its spacial and agricultural limitations, probably has one of the most constant, restricted environments of any human population.

Figure 2 shows the increase in the gene frequency variance, and these increases parallel the decreases in the phenotypic variance. The maximum variance would be 0.25 with $50 \%$ of the loci fixed for the minus allele and $50 \%$ for the plus allele. After 100 generations, the populations of 25 are close to the maximum. For the runs without selection these increases in gene frequency variance and the decreases in phenotypic variance can be compared to their theoretical expectations. The rate of increase in gene frequency variance is proportional to $1 / 2 \mathrm{~N}$, so that after $\mathrm{t}$ generations the variance would be: $\mathrm{V}_{\mathrm{t}}=$ $\mathrm{p}(1-\mathrm{p})\left[1-(1-1 / 2 \mathrm{~N})^{\mathrm{t}}\right]$ (Crow and Kimura, $70: 328)$. After 100 generations one would expect variances of $0.2163,0.1585$, and 0.0825 for populations of 25,50 , and 100 , respectively. The averages of the runs with no selection on table 2 are $0.1934,0.1275$, and 0.0919 , which are close to the expected values although in some cases they are significantly different. For any run the S.D./ $\sqrt{10}$ can provide an approximate significance test. In all cases selection has increased the rate of change in the gene frequency variance, which has resulted in larger variances and some which are closer to the theoretical expectations.

The general formula for the decline in the genotypic variance is: $V_{t}=V_{0} e^{-t / 2 N}$ (Crow and Kimura, '70:340). After 100 generations the variance should thus be $13.5 \%, 36.6 \%$, and $66 \%$ of the original variance for populations of 25,50 , and 100 , respectively. On our scale all runs were started with the same allelic frequencies and hence with the same theoretical phenotypic variance of about 0.0425 . Since there is no environmental component, the genotypic and phenotypic variances are the same. After 100 generations the expected variances would be: $0.00574,0.01555$, and 0.02805 for populations of 25,50 , and 100 , respectively. The averages of the actual runs with no selection are: $0.0060,0.0192$, and 0.0262 . As in the case of the gene frequency variance, stabilizing selection has increased the rate of change, so that the phenotypic variance is smaller in the runs with selection.

\section{DISCUSSION}

The simulations reported here thus seem to be in reasonable agreement with the theoretical expectations of the effects of drift on metric traits. They also show that stabilizing selection, which seems to be operating in human populations, does not counteract drift but instead reinforces it. It has been known for some time (e.g., Haldane, '32) that stabilizing selection for a metric trait does not result in stable polymorphic gene frequencies. If loci with equal and additive effects is an appropriate model for stature and other human metric traits, then a considerable amount of homozygosity would be expected, not only in small populations due to drift but in large ones due to selection. However, the maintenance of variability in small populations and the fact that hybrid populations are intermediate for most traits (Trevor, '53) seem to be evidence against much homozygosity and in addition evidence against the widespread occurrence in human populations of the evolutionary process which has been simulated in this paper. 


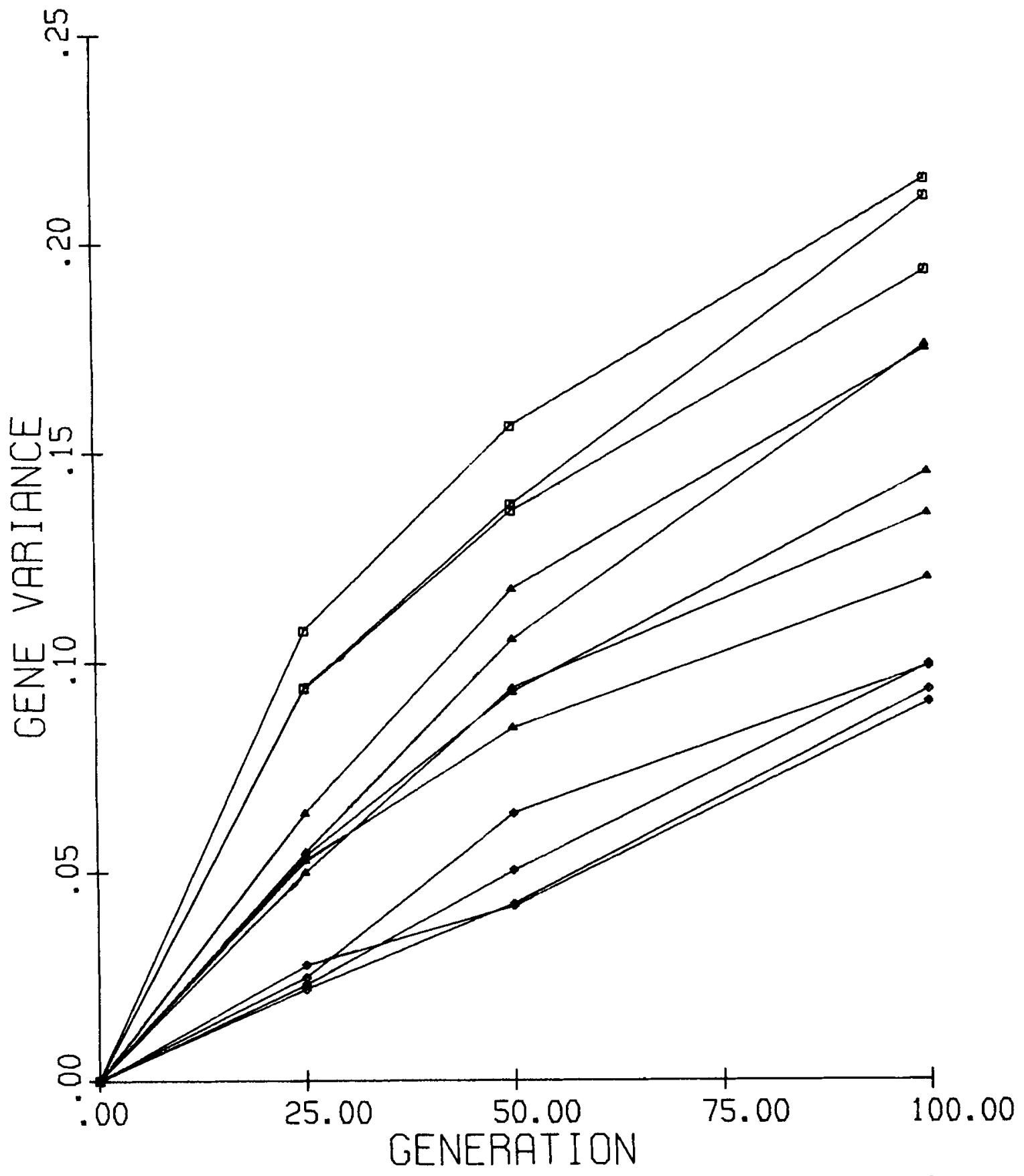

Fig. 2 The increase in the gene frequency variance with different population sizes and with different amounts of optimizing selection. The runs shown here are the same as those in

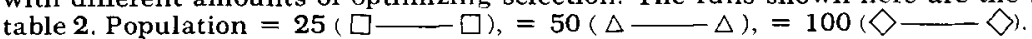


The question then arises as to why the model does not seem to accord with the data on human metric traits. There are several possible answers. Robertson ('56) has shown that if heterozygosity per se is assumed to increase fitness, then a stable polymorphic equilibrium would exist for all loci. Heterozygote superiority involves further assumptions as to the nature of gene action and as to the determination of genotype fitness. But if the fitness of the genotype is determined primarily by the phenotype, which seems to be the most reasonable and simplest assumption, then Robertson's model would not seem to be as appropriate for quantitative traits. On the other hand, Kojima ('59) showed that with almost complete dominance and the optimum phenotype close to the maximum a stable polymorphic equilibrium could exist for two loci. Lewontin ('64) extended Kojima's results to $\mathbf{n}$ loci, and Singh and Lewontin ('66) showed how the area of stability was extended by linkage. However, Gale and Kearsey ('68a) pointed out that this model requires the dominance to be uni-directional and equal for all loci, and this is not true for traits in Drosophila and probably not for most human traits. Instead they show that unequal effects for additive loci can also lead to stable polymorphic equilibria. For a trait determined by three loci Gale and Kearsey ('68b) have shown that with stabilizing selection which has the fitness of the genotype vary linearly with its distance from the optimum, it is possible to have polymorphic equilibria with no linkage. If, instead of all allelic substitutions contributing 1.0 units to the genotype as in our model, the three loci contribute $0.17,1.0$, and 1.79 units, then there will be stable polymorphic equilibria for all three loci. If the allelic effects are more equal than these values, then selection will tend to increase homozygosity. Although the differences in these effects are quite large, the smallest being five times less than the median and the largest twice as great, this model can probably explain some of the quantitative variation in human populations. Gale and Kearsey's model is not the usual quadratic optimum model, but Bulmer (71) has shown that any optimum model with equal and additive effects will not have a stable equilibrium with polymorphic frequencies at all loci.

Kimura ('65) has presented a rather different model for the maintenance of quantitative variation which includes mutational effects. In fact, in his model mutation is the major force maintaining genetic variation, while stabilizing selection decreases it. Selection is proportional to the effect of the allele, so that mutation at a high enough rate may be able to maintain several alleles with small differences in their effects in a population. These would act as "neutral" mutations, and it would not seem likely that the genetic variation in most human metric traits, such as stature, is neutral or nonadaptive. Nevertheless, for similar reasons Allen ('70) has suggested that the genetic variation in IQ not associated with mental deficiency may be non-adaptive. The most realistic model for metric traits may thus be comparable to recent models of amino acid evolutionary changes in proteins. Some amino acid substitutions seem to have little biochemical or phenotypic effect and thus are neutral and influenced by drift and mutation to such an extent that many polymorphisms would be expected, while other substitutions with major phenotypic effects seem to be controlled by selection (King and Jukes, '69). Normal variation in metric traits would also seem likely to be due to both kinds of allelic substitutions.

\section{LITERATURE CITED}

Allen, G. 1970 Within group and between group variation expected in human behavioral characters. Behavior Genetics, 1 : 175-194.

Bajema, C. J., ed. 1971 Natural Selection in Human Populations. John Wiley and Sons, New York.

Barrai, I, L. L. Cavalli-Sforza and M. Mainardi 1964 Testing a model of dominant inheritance for metric traits in man. Heredity, 19: 651-668.

Birdsell, J. B. 1950 Some implications of the genetical concept of race in terms of spatial analysis. Cold Spring Harbor Symposia in Quantitative Biology, 15 : 259-314.

Bulmer, M. G. 1971 The stability of equilibria under selection. Heredity, 27: 157-162.

Crow, J. F., and M. Kimura 1970 An Introduction to Population Genetics Theory. Harper and Row, New York.

Curnow, R. N. 1964 The effect of continual selection of phenotypic intermediates on gene frequency. Genetical Research, 5: 341-353.

Fisher, R. A. 1918 The correlation between relatives on the supposition of Mendelian in- 
heritance. Transactions of the Royal Society of Edinburgh, 52: 399-433.

Frankham, R., L. P. Jones and J. S. F. Barker 1968 The effects of population size and selection intensity in selection for a quantitative character in Drosophila. Genetical Research, 12: 237-248.

Gale, J. S., and M. J. Kearsey 1968a Stable equilibria under stabilising selection in the absence of dominance. Heredity, 23: 553-561.

$1968 \mathrm{~b}$ Stable equilibria under stabilising selection in the absence of dominance: an additional note. Heredity, 23: 617-620.

Gomila, J. 1971 Les Bedik. University of Montreal Press, Montreal.

Haldane, J. B. S. 1932 The Causes of Evolution. Harper and Row, New York.

Harrison, G. A. 1971 The application of spectrophotometry to studies of skin color in Latin American populations. In: The Ongoing Evolution of Latin American Populations. F. M. Salzano, ed. Charles C Thomas, Springfield, Illinois, pp. $455-469$

Hill, W. G. 1969 Artificial selection in finite populations. Genetical Research, $13: 143-164$.

Howells, W. W. 1936 Some uses of the standard deviation in anthropometry. Human Biology, 8: 592-600.

Kimura, M. 1965 A stochastic model concerning the maintenance of genetic variability in quantitative characters. Proc. National Academy Sciences, 54: 731-736.

King, J. L., and T. H. Jukes 1969 Non-Darwinian evolution. Science, 164: 788-798.

Kojima, K-I. 1959 Stable equilibria for the optimum model. Proc. National Academy Sciences, 45: 989-993.

Latter, B. D. H. 1970 Selection in finite populations with multiple alleles. II. Centripetal selection, mutation, and isoallelic variation. Genetics, 66: 165-186.

Latter, B. D. H., and C. E. Novitski 1969 Selection in finite populations with multiple alleles. I. Limits to directional selection. Genetics, 62: 859-876,
Lewontin, R. C. 1964 The interaction of selection and linkage. II. Optimum models. Genetics, 50: 757-782.

Livingstone, F. B. 1969 Polygenic models for the evolution of human skin color differences. Human Biology, 41: 480-493.

Marshall, W. A., J. M. Tanner, H. E. Lewis and M. A. Richardson 1971 Anthropometric measurements of the Tristan da Cunha Islanders 1962-1968. Human Biology, 43: 112-139.

Penrose, L. S. 1969 Effects on additive genes at many loci compared with those of a set of alleles at one locus in parent-child and sib correlations. Annals of Human Genetics, 33: 15-21.

Prout, T. 1962 The effects of stabilizing selection on the time of development in Drosophila melanogaster. Genetical Research, 3: 364-382.

Robertson, A. 1956 The effect of selection against extreme deviants based on deviation or on homozygosis. Journal of Genetics, 54: 236-248.

Scharloo, W. M. S. Hoogmoed and A. ter Kuile 1967 Stabilizing and disruptive selection on a mutant character in Drosophila. I. The phenotypic variance and its components. Genetics, 56: 709-726.

Singh, M., and R. C. Lewontin 1966 Stable equilibria under optimizing selection. Proc. National Academy Sciences, 56: 1345-1348.

Spuhler, J. N. 1968 Sociocultural and biological inheritance in man. In: Genetics. D. V. Glass, ed. Rockefeller University Press, New York, pp. 102-110.

Trevor, J. C. 1953 Race Crossing in Man. Eugenics Laboratory Memoirs No. 36. University of London Press, London.

Young, S. S. Y. 1966 Computer simulation of directional selection in large populations. I. The programme, the additive and the dominance models. Genetics, 53: 189-205.

- 1967 Computer simulation of directional selection in large populations. II. The additive $\times$ additive and mixed models. Genetics, 56: 73-87. 